\title{
Knot in Cen A: Stochastic Magnetic Field for Diffusive Synchrotron Radiation?
}

\author{
Jirong Mao and Jiancheng Wang \\ Yunnan Observatory, National Astronomical Observatories, Chinese Academic of Sciences, \\ P.O. Box 110, Kunming, Yunnan Province, 650011, China \\ jirongmao@ynao.ac.cn
}

\begin{abstract}
The emission of relativistic electrons moving in the random and small-scale magnetic field is presented by diffusive synchrotron radiation (DSR). In this Letter, we revisit the perturbative treatment of DSR. We propose that random and small-scale magnetic field might be generated by the turbulence. As an example, multi-band radiation of the knot in Cen A comes from the electrons with energy $\gamma_{e} \sim 10^{3}-10^{4}$ in the magnetic field of $10^{-3} \mathrm{G}$. The multi-band spectrum of DSR is well determined by the feature of stochastic magnetic field. These results put strong constraint to the models of particle acceleration.
\end{abstract}

Subject headings: radiation mechanisms: nonthermal — galaxies: jet — galaxies: active - galaxies: individual (Cen A) - turbulence

\section{Introduction}

A popular explanation of non-thermal emission from objects such as Gamma-Ray Burst (GRB) and jet in Active Galactic Nuclei (AGN) is synchrotron radiation. The relativistic electrons are buried in the external, homogenous and steady magnetic field. However, this large-scale magnetic field is a priori and the origin of it in GRBs and jets is under debate. Alternatively, the perturbative and more general non-perturbative treatments of Diffusive Synchrotron Radiation (DSR) have been proposed (Toptygin \& Fleishman 1987; Fleishman 2006a), the so-called jitter radiation is a specific limiting 1D case within the general perturbative DSR theory. DSR is the emission of relativistic electrons in the local and random magnetic field. The magnetic field might be produced by the following process: the anisotropic-distributed plasma can be disturbed by relativistic collisionless shocks, hence, the initial magnetic field is produced by the perturbation. The induced currents from the mag-

netic field amplify the original magnetic field thus Weibel instability occurs (Weibel 1959; 
Medvedev \& Loeb 1999; Frederiksen et al. 2004; Hededal \& Nishikawa 2005). Due to the lack of the external magnetic field, the particle acceleration can not be treated by the Feimiacceleration (Hededal et al. 2004; Nishikawa et al. 2006) as the usual way. The DSR and jitter radiation have been selected to predict the spectrum of GRB/afterglow (Fleishman 2006a; Medvedev 2006; Medvedev et al. 2007; Workman et al. 2007) and knot in the jet (Fleishman 2006b). These analytical results are also identified with numerical simulations (e.g...Hededal \& Nordlund 2006).

There are still some problems which should be concentrated on. For instance, the plasma frequency $\omega_{p e}=\left(4 \pi e^{2} n / m_{e}\right)^{1 / 2}$, as a function of the electron density $n$ in the plasma, is introduced, thus the radiation properties are strongly affected by the local environment. Medvedev et al. (2005) played with the model by merging current filaments to generate the magnetic field, while in principle the generation of the magnetic field should be linked with the perturbation of the fluid field. Besides this filaments merging effect, there could be other possibilities to produce random magnetic field.

In this paper, following the previous work of Fleishman (2006a) and Medvedev (2006), we put forward the case of emission by relativistic electrons moving in stochastic magnetic field. In Section 2, we review the perturbative DSR and focus on the origin of the magnetic field. The local and random magnetic field may be produced by turbulence, but not Weibel instability. In Section 3, we compare our results to the multi-band spectrum of the knot in Centaurus A (hereafter Cen A). Finally, the discussion and future expectation are given in Section 4.

\section{Radiation Revisited and Stochastic Magnetic Field}

The emission of single relativistic particle in the small-scale magnetic field was firstly introduced by Landau \& Lifshitz (1971). Here, we follow the developed formula to calculate the radiation intensity, which is the energy per unit frequency per unit time (Fleishman 2006a):

$I_{\omega}=\frac{e^{4}}{m^{2} c^{3} \gamma^{2}} \int_{1 / 2 \gamma_{*}^{2}}^{\infty} d\left(\frac{\omega^{\prime}}{\omega}\right)\left(\frac{\omega}{\omega^{\prime}}\right)^{2}\left(1-\frac{\omega}{\omega^{\prime} \gamma_{*}^{2}}+\frac{\omega^{2}}{2 \omega^{\prime 2} \gamma_{*}^{4}}\right) \times \int d q_{0} d \mathbf{q} \delta\left(\omega^{\prime}-q_{0}+\mathbf{q v}\right) K(\mathbf{q}) \delta\left(q_{0}-q_{0}(\mathbf{q})\right)$

where $\omega^{\prime}=(\omega / 2)\left(\gamma^{-2}+\theta^{2}+\omega_{p e}^{2} / \omega^{2}\right), \theta$ is the angle between the electron velocity and the radiation direction, $\mathbf{q}$ and $q_{0}$ are the wave number and frequency of the disturbed field respectively, $\gamma_{*}^{-2}=\gamma^{2}+\omega_{p e}^{2} / \omega^{2}$ and $\gamma$ is the electron energy, $K(\mathbf{q})$ is the term for the random magnetic field. 
Equation (1) is the general expression for perturbative treatment. It is pointed out by Fleishman (2006a), the rectilinear motion of electron is valid for large frequencies, however, at low frequencies, the particle trajectory traverses several correlation lengths scattering by magnetic inhomogeneities, thus the particle deflection angle accumulated along the coherence length exceeds the beaming angle (see Figure 1 of Fleishman (2006a)).

The dispersion relation $q_{0}=q_{0}(q)$ of the non-relativistic plasma was presented in Weibel (1959). The improved equations for the isotropic and relativistic plasma were given in detail by Mikhailovski (1980) while Yoon \& Davidson (1987) built the analytical model for the relativistic plasma with a waterbag distribution. More comprehensive works have been performed recently by Silva et al. (2002), Wiersma \& Achterberg (2004) and Fiore et al. (2006). In this paper, we choose the dispersion relation of relativistic collisionless shocks considered by Milosavljević, Nakar \& Spitkovsky (2006).

Weibel instability is an efficient way to generate the random magnetic field in relativistic shocks (Silva et al. 2003; Schlickeiser \& Shukla 2003; Wiersma \& Achterberg 2004; but see also Lyubarsky \& Eichler 2006). However, there could be other possibilities to form magnetic structure. In this Letter, we argue that the local and random magnetic field generated by turbulence is also relevant for the perturbative DSR theory.

The spectrum energy in a fully developed turbulent fluid can be described by the Kolmogorov form with the classical index $-5 / 3$. For the magnetic turbulence, the cascade delay time may enter the estimation of energy transfer rate, the energy spectrum of Kraichnan has an index of $-3 / 2$. Although the situation we focus on has no external magnetic field, at small scales, the turbulence is still shown as the cascade properties. Self-excited Alfvén turbulence has also been found (Sokolov et al. 2006). Moreover, we note that the non-magnetized and magnetized turbulence have a high-degree similarity (Cho, Lazarian \& Vishniac 2002; Lazarian \& Beresnyak 2004). All these evidences indicate that a general form of fluid turbulence can also be valid for the study of random magnetic field generation. Furthermore, the index of the turbulence spectrum is not universal. Zhou \& Matthaeus (1990) investigated local turbulent effects with transport models and other nonlinear terms. Using the scaling model (She \& Leveque 1994) which presents the cascade as an infinitely-divisible log-Poisson process (She \& Waymire 1995), Boldyrev, Nordlund \& Padoan (2002) derived a steeper spectrum compared to that of Kolmogorov. In fact, as estimated by Wang (2002), the index value of a turbulent spectrum has the range between -1 to -2 . MacLow \& Ossenkopf (2000) found that the local turbulent spectrum does not show a straight power-law. Therefore, we propose that the turbulent spectrum be shown as:

$$
F(k) \propto k^{-\alpha} f\left(k / k_{\eta}\right)
$$

where $k_{\nu}<k<k_{\eta}, k_{\nu}$ corresponds to the viscous scale of the fluid while $k_{\eta}$ is linked with 
the scale of the resistive cascade transfer. We choose $f\left(k / k_{\eta}\right)$ as an exponential-drop form.

The magnetic field amplified by the turbulence spectrum has been described by Niemiec \& Ostrowski (2004, 2006). We obtain the amplified magnetic field as:

$$
\left\langle\delta B^{2}(k)\right\rangle \propto \int_{k}^{\infty} F\left(k^{\prime}\right) d k^{\prime}
$$

In general, Eq.(2) presented as a power-law with a cutoff at high wave number is universal for the fluid dynamo turbulence, whatever the radiation field is. The $K(\mathbf{q})$ in Eq.(1) can be linked by the magnetic field as $K(\mathbf{q})=C_{0}\left\langle\delta B^{2}(q)\right\rangle$ where $C_{0}$ is the normalization number. Therefore, this turbulent approach for obtaining magnetic field is the developments in the framework of current DSR theory.

\section{The Case of Cen A}

Cen A, the nearest proto-FRI galaxy, was sketched from the observational view (Israel 1998). In particular, the knot in the jet has been detected in radio, X-ray (Hardcastle et al. 2003; Kraft et al. 2003; Kataoka et al. 2006) and infrared (Hardcastle, Kraft \& Worrall 2006) bands. With these observations, this object provides a multi-band spectrum to constrain the radiation mechanisms and the models of particle acceleration.

The central density of the knot in Cen $\mathrm{A}$ is $n=3.7 \times 10^{-2} \mathrm{~cm}^{-3}$ (Kraft et al. 2003), the correlation length of the random magnetic field is estimated by $l_{c o r} \sim(0.1-1) l_{s k} \sim$ $10^{5}-10^{6} \mathrm{~cm}$ where $l_{s k}=c / w_{p e}$ is the skin depth, while the size of the knot is less than $10 \mathrm{pc}$ (Hardcastle et al. 2003). The flare points and complicated light curves (Hardcastle, Kraft \& Worrall 2006) indicate the disturbed effects of the irregular magnetic fields. These small-scale random inhomogeneities give us the opportunity to calculate the emission using perturbative DSR. We insert Eq.(2) to Eq.(1) and calculate numerically, we set the turbulent spectrum $\alpha=1.45$. The electron energy distribution $d N / d \gamma \propto \gamma^{-s}$ is assumed as $s=3.3$. The bulk Lorenz factor is $\Gamma=12$. The range of $k$ for turbulent spectrum calculation can be estimated by $k_{\eta} / k_{\nu}=P_{r}^{1 / 2}$, where the Prandtl number is $P_{r} \sim 10^{-5} T^{4} / n \sim 10^{14}$ for the warm medium in the knot of Cen A (Schekochihin \& Cowley 2007). The final result with the comparison to the observational data is shown in Fig. 1. Thus, we use the single gross turbulent spectrum to reproduce the multi-band emission, with its drop-off point properly shown in the X-ray band. From the data fitting, we find that the relativistic electrons with $1 \leq \gamma_{e} \leq 10^{3}-10^{4}$ are enough for this multi-band emission, while the turbulent magnetic field is strong, at least $10^{-3} \mathrm{G}$, which is larger than the equipartition value of $100 \mu \mathrm{G}$ estimated by synchrotron radiation (Kataoka et al. 2006). 
The radiative cooling of synchrotron emission may be one of the reasons to explain the deeper spectrum toward high energy bands (Heavens \& Meisenheimer 1987; Meisenheimer et al. 1989). The observation of M87 supports this traditional interpretation (Harris et al. 2006$)$. And the synchrotron emission by two population of electrons is needed (Sambruna et al. 2001) to explain the X-ray spectrum of 3C273. But for the spectrum of the knot in Cen A, the difference of the spectral indexes between the flatter part and the deeper part is less than 0.5 (Hardcastle, Kraft \& Worrall 2006). This is contradictory to the prediction of typical synchrotron electron cooling. For another point of view, the relatively low number density of the knot can contribute just a small amount of absorption, thus the strong decrease of flux in X-ray band is not due to dust attenuation. Therefore, the drop-off point in the spectrum might present the behavior of the turbulence.

From another side, we may directly describe the magnetic field as: $B^{2}(k) \propto k^{-p}$. For this point, we avoid the detailed treatments of any turbulence model. With the double power-law as the form of magnetic field to calculate DSR, we select $p_{1}=1.4$ and $p_{2}=1.7$ respectively to get the result shown in the Fig. 2. But the bulk Lorenz factor is changed from $\Gamma_{1}=12$ to $\Gamma_{2}=2$. This result gives us an alternative clue to explain the multi-band spectrum: the break point in the spectrum might indicate the bulk transition state of the shock from extra-relativistic to sub-relativistic phase.

There are some knots in other objects observed by multi-band telescopes. Different knots have different spectral slopes and different quantities of flux, indicating the non-uniform turbulent mode and different acceleration processes. In this paper, we give the example of Cen A. However, whatever the spectral shape is, we see that the observational spectrum can be explained by perturbative DSR theory, the emission is dominated by the random magnetic field which could be amplified by the turbulence. Thus, the spectral shape is uniquely determined by the random magnetic field from radio to X-ray band.

\section{Discussion}

In this paper, we use the turbulent spectrum to amplify the random magnetic field. We find that the spectrum shape of DSR is only dominated by the stochastic magnetic field. The existence of this kind of magnetic field has been confirmed by numerical simulations (Haugen, Brandenburg \& Dobler 2004a,b; Schekochihin et al. 2004). Thus, the whole multiband radiation is produced originally from a relatively small region, about several $p c$, with a series of physical processes.

Furthermore, the light curves at radio, infrared and X-ray band of the knots are more 
complicated. It seems that the emission is firstly seen in the X-ray band, then followed by infrared and radio bands (Hardcastle, Kraft \& Worrall 2006). We expect that the turbulent magnetic field could have time evolution during the cascade process with energy transfer. The final radiation spectrum may be a composite result from the multi-structure of the turbulence spectra and is averaged by the time evolution. Deep research of the delicate structure in the turbulent magneto-fluid is encouraged to explain the time-dependent features.

Three aspects are included in the whole scenario: turbulence, magnetic field and particle acceleration. In our opinion, firstly, the fluid background is disturbed by the relativistic collision-less shock, the perturbative dynamos are distributed as the turbulence spectrum; then, the initial magnetic elements are amplified by the turbulence, shown as the random and small-scale magnetic field; finally, the particles can be accelerated by relativistic shocks and/or turbulent flow to produce DSR as a first step, then continually accelerated to the higher energy part by other mechanisms which are related to the mature magnetic field. For simplicity, in this Letter, we assume that the electron injection is continuous so that the spectrum does not show energy loss by radiation.

The electron energy distribution $d N(\gamma) / d \gamma \propto \gamma^{-s}$ has no universal index $s$ (Shen, Kumar \& Robinson 2006). This suggests that particle acceleration may also have multiple processes. There are at least two ways to accelerate electrons. Honda \& Honda (2005) considered that the electrons are accelerated by the interaction with the local magnetic filaments; although our model prohibits an external magnetic field, since the Alfvén turbulence can be self-excited by the diffusive shocks (Sokolov et al. 2006), the popular Fermi and stochastic acceleration can also be accepted in the local region. Other models reveal that the index $s$ varies with the upstream and downstream of the shock (Keshet \& Waxman 2005; Baring 2007). Recent research even finds that particle acceleration is affected by the equation of state (Morlino, Blasi \& Vietri 2007). Further investigation into the relationship of turbulence, magnetic field and particle acceleration would be expected.

We thank A. Celotti and F. Yuan for the helpful discussion. This work is financially supported by the Chinese National Science Fund 10673028.

\section{REFERENCES}

Baring, M. G. Ap\&SS, 307, 297

Boldyrev, S., Nordlund, Å., \& Padoan, P. 2002, Phys. Rev. Lett., 89, 1102

Cho, J., Lazarian, A., \& Vishniac E. T. 2002, ApJ, 566, L49 
Fiore, M., Silva, L. O., Ren, C., Tzoufras, M. A., \& Mori, W. B. 2006, MNRAS, 372, 1851

Fleishman, G. D. 2006a, ApJ, 638, 348

Fleishman, G. D. 2006b, MNRAS, 365, L11

Frederiksen, J. T., Hededal, C. B., Haugbølle, T., \& Nordlund, Å. 2004, ApJ, 608, L13

Hardcastle, M. J., Worrall, D. M., Kraft, R. P., Forman, W. R., Jones, C., \& Murray, S. S. 2003, ApJ, 593, 169

Hardcastle, M. J., Kraft, R. P., \& Worrall, D. M. 2006, MNRAS, 368, L15

Harris, D. E., et al. 2006, ApJ, 640, 211

Haugen, N. E. L., Brandenburg, A., \& Dobler, W. 2004a, Ap\&SS, 292, 53

Haugen, N. E. L., Brandenburg, A., \& Dobler, W. 2004b, Phys. Rev. E., 70, 016308

Heavens, A. F., \& Meisenheimer, K. 1987, MNRAS, 225, 335

Hededal, C. B., Haugbølle, T., Frederiksen, J. T., \& Nordlund, Å. 2004, ApJ, 617, L107

Hededal, C. B., \& Nishikawa, K.-I. 2005, ApJ, 623, L89

Hededal, C. B., \& Nordlund, Å. 2006, ApJ, preprint: astro-ph/0511662

Honda, M., \& Honda, Y. 2005, ApJ, 633, 733

Israel, F. P. 1998, A\&ARev., 8, 237

Kataoka, J., Stawarz, L., Aharonian, F., Takahara, F., Ostrowski, M., \& Edwards, P. G. 2006, ApJ, 641, 158

Keshet, U., \& Waxman, E. 2005, Phys. Rev. Lett., 94, 1102

Kraft, R. P., et al. 2003, ApJ, 592, 129

Landau, L. D. \& Lifshitz, E. M. 1971, The classical theory of fields, Pergamon Press, Oxford

Lazarian, A. \& Beresnyak, A. 2004, Proceedings of the Magnetized Plasma in Galaxy Evolution, Poland, preprint: astro-ph/0505577

Lyubarsky, Y. \& Eichler, D. 2006, ApJ, 647, 1250

MacLow, M.-M., \& Ossenkopf, V. 2000, A\&A, 353, 339 
Medvedev, M. V. \& Loeb, A. 1999, ApJ, 526, 697

Medvedev, M. V., Fiore, M., Fonseca, R. A., Silva, L. O., \& Mori, W. B. 2005, ApJ, 618, L75

Medvedev, M. V. 2006, ApJ, 637,869

Medvedev, M. V., Lazzati, D., Morsony, B. C., \& Workman, J. C. 2007, ApJ, preprint: astro-ph/0703209

Meisenheimer, K. et al., 1989, A\&A, 219, 63

Mikhailovski, A. B. 1980, Plasma Phys, 22, 133

Milosavljević, M., Nakar, E., \& Spitkovsky, A. 2006, ApJ, 637, 765

Morlino, G., Blasi, P., \& Vietri, M. 2007, ApJ, preprint: astro-ph/0703555

Niemiec, J., \& Ostrowski, M. 2004, ApJ, 610, 851

Niemiec, J., \& Ostrowski, M. 2006, ApJ, 641, 984

Nishikawa, K.-I., Hardee, P. E., Hededal, C. B., \& Fishman, G. J. 2006, ApJ, 642, 1267

Sambruna, R. M., et al. 2001, ApJ, 549, L161

Schekochihin, A. A., Cowley, S. C., Taylor, S. F., Maron, J. L. \& McWilliams, J. C. 2004, ApJ, 612, 276

Schekochihin, A. A., \& Cowley, S. C. 2007, invited chapter in Magnetohydrodynamics: Historical evolution and trends, Berlin: Springer, preprint: astro-ph/0507686

Schlickeiser, R., \& Shukla, P. K. 2003, ApJ, 599, L57

She, Z.-S., \& Leveque, E. 1994, Phys. Rev. Lett., 72, 336

She, Z.-S., \& Waymire, E. C. 1995, Phys. Rev. Lett., 74, 262

Shen, R., Kumar, P., \& Robinson, E. L. 2006, MNRAS, 371, 1441

Silva, L. O., Fonseca, R. A., Tonge, J. W., Mori, W. B., \& Dawson, J. M. 2002, Phys. Plasmas, 9, 2458

Silva, L. O., Fonseca, R. A., Tonge, J. W., Dawson, J. M., Mori, W. B. \& Medvedev, M. V. 2003, ApJ, 596, L121 
Sokolov, I. V., Roussev, I. I., Fisk, L. A., Lee, M. A., Gombosi, T. I., \& Sakai, J. I. 2006, ApJ, 642, L81

Toptygin, I. N. \& Fleishman, G. D. 1987, Ap\&SS, 132, 213

Wang, J. 2002, Chin. J. Astro. Astrophys., 2, 1

Weibel, E. S. 1959, Phys. Rev. Lett., 2, 83

Wiersma, J. \& Achterberg, A. 2004, A\&A, 428, 365

Workman, J. C., Morsony, B. C., Lazzati, D., \& Medvedev, M. V. 2007, MNRAS, preprint: astro-ph/0703420

Yoon, P. H. \& Davidson, R. C. 1987, Phys. Rev. A., 35, 2718

Zhou, Y., \& Matthaeus, W. H. 1990, J. Geophys. Res., 95, 14881

This preprint was prepared with the AAS IATEX macros v5.2. 


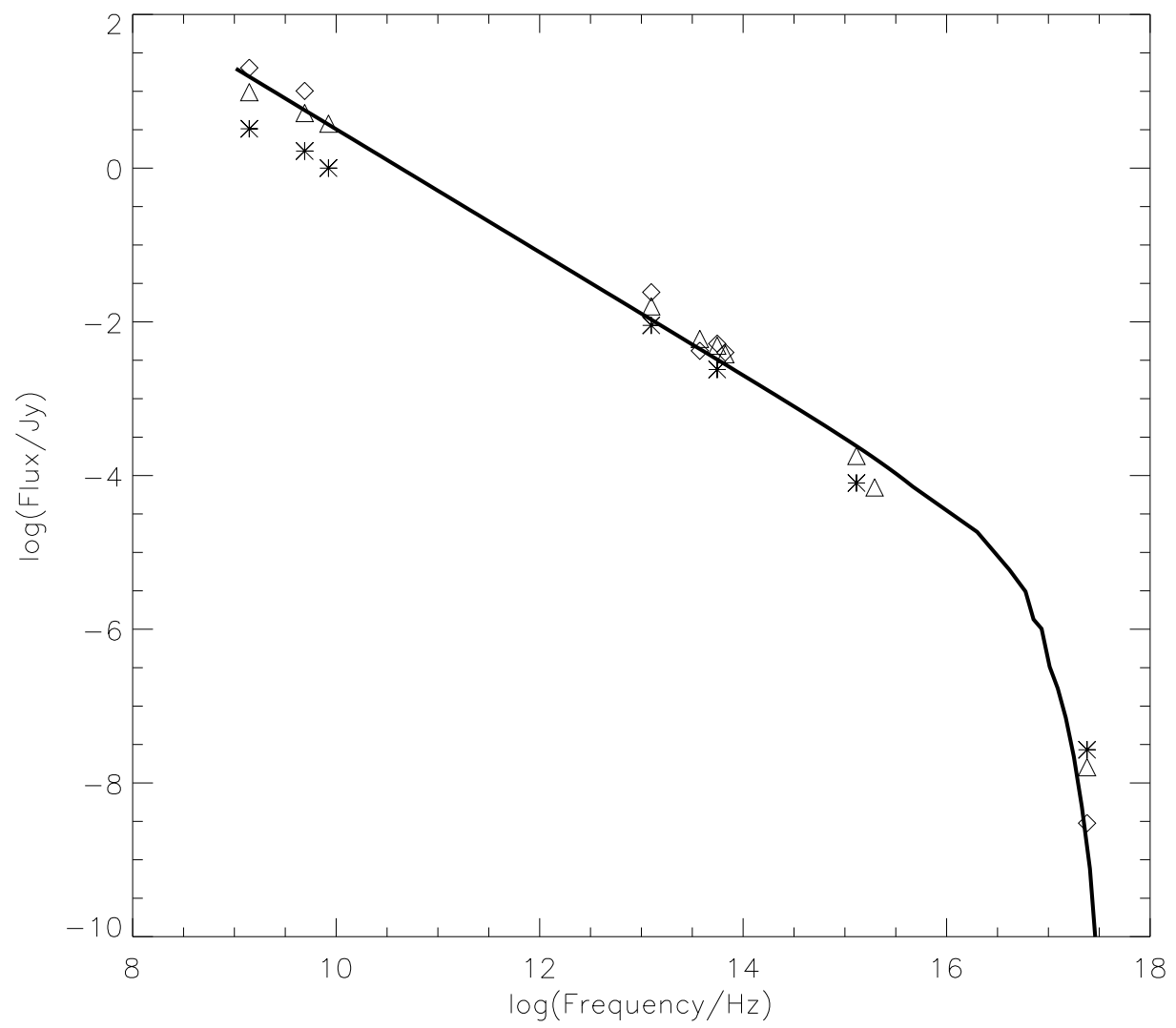

Fig. 1. - Multi-band spectrum of knot in Cen A predicted by perturbative DSR. Magnetic field is obtained by the Eq. (3). The observational data (inner, middle and outer regions are symbolized by stars, triangles and diamonds respectively) are collected from Hardcastle, Kraft \& Worrall (2006). Our prediction is denoted as the solid line. 


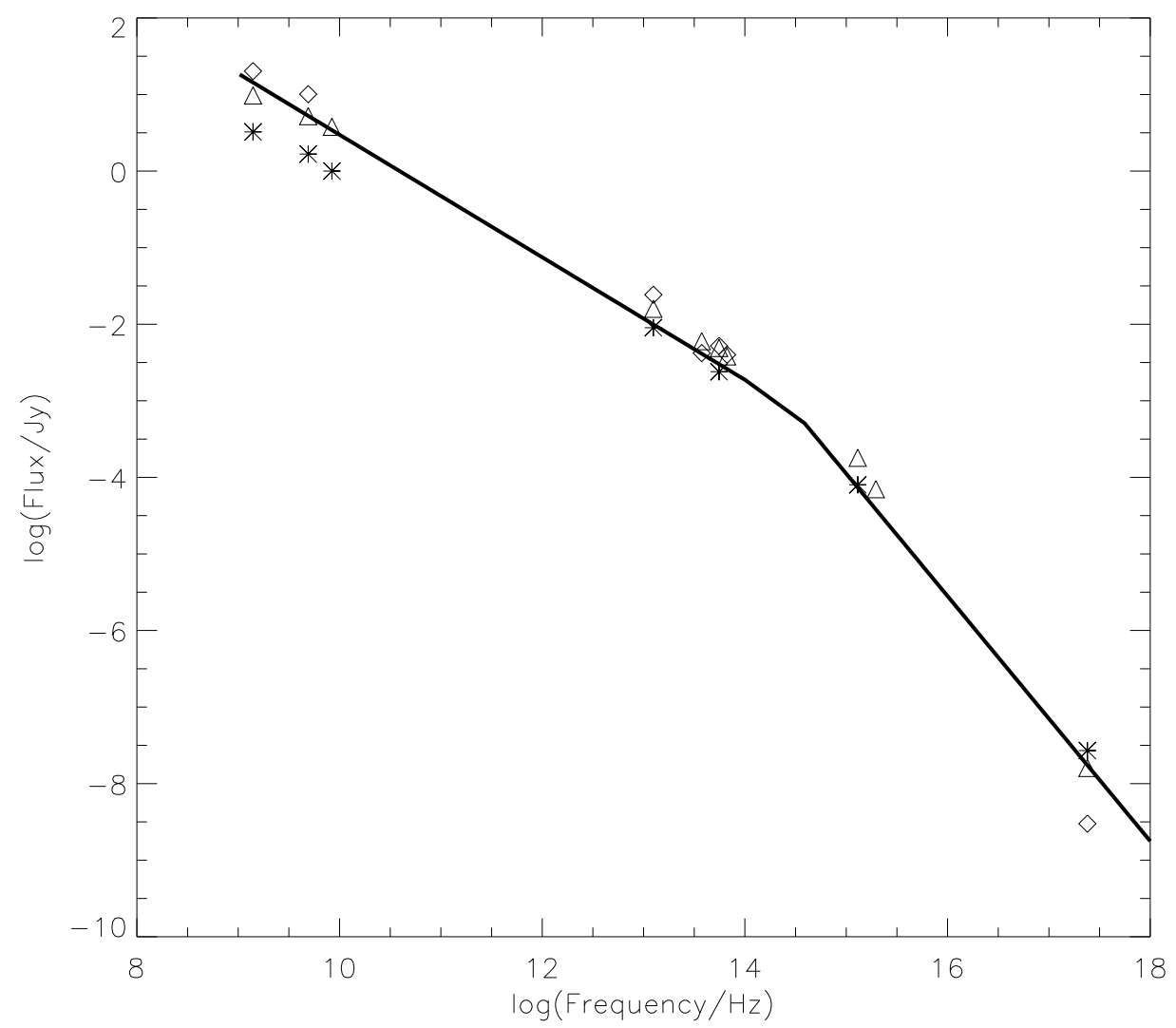

Fig. 2.- Multi-band spectrum of knot in Cen A predicted by perturbative DSR. The solid line denotes the radiation determined directly by the random magnetic field of a double power-law. The observational data are shown as same as those in the Fig.1. 